\title{
Anterior cruciate ligament tear induces a sustained loss of muscle fiber force production
}

Jonathan P Gumucio, $\mathrm{PhD}^{1,2}$, Kristoffer B Sugg, MD, PhD ${ }^{1,2,3}$, Elizabeth R Sibilsky Enselman $\mathrm{MEd}^{1}$, Alexis C Konja, BS ${ }^{1}$, Logan R Eckhardt ${ }^{1}$, Asheesh Bedi, MD ${ }^{1}$, Christopher L Mendias, $\mathrm{PhD}^{1}$

Departments of ${ }^{1}$ Orthopaedic Surgery, ${ }^{2}$ Molecular \& Integrative Physiology, and ${ }^{3}$ Surgery, Section of Plastic Surgery, University of Michigan Medical School, Ann Arbor, MI

${ }^{4}$ Hospital for Special Surgery, New York, NY

*To whom correspondence should be addressed:

Christopher L Mendias, PhD

Hospital for Special Surgery

535 E 70th St

Research Institute - S.602

New York, NY 10021

MendiasC@hss.edu

917-260-3783

Running Title: Muscle Fiber Dysfunction After ACL Tear

Acknowledgements: This work was supported by NIH grants F31-AR065931 and F32-

AR067086. The authors would like to acknowledge technical assistance from Dr. Dennis Claflin, Mr. Dylan Sarver, and Mr. Christopher Ciric.

Number of Words in Abstract: 148

Number of Words in Manuscript: 1477

Conflict of Interest: None of the authors have any conflict of interest to disclose.

Ethical Publication Statement: We confirm that we have read the Journal's position on issues involved in ethical publication and affirm that this report is consistent with those guidelines.

This is the author manuscript accepted for publication and has undergone full peer review but has not been through the copyediting, typesetting, pagination and proofreading process, which may lead to differences between this version and the Version of record. Please cite this article as doi:10.1002/ mus.26075. 


\section{Abstract}

Introduction: Patients with anterior cruciate ligament (ACL) tears have persistent quadriceps strength deficits that are thought to be due to altered neurophysiological function. Our goal was to determine the changes in muscle fiber contractility independent of the ability of motor neurons to activate fibers.

Methods: We obtained quadriceps biopsies of patients undergoing ACL reconstruction, and additional biopsies one, two, and six months after surgery. Muscles fiber contractility was assessed in vitro, along with whole muscle strength testing.

Results: Compared to controls, patients had a $30 \%$ reduction in normalized muscle fiber force at the time of surgery. One month later the force deficit was $41 \%$, and at 6 months the deficit was $23 \%$. Whole muscle strength testing demonstrated similar trends.

Discussion: While neurophysiological dysfunction contributes to whole muscle weakness, there is also a reduction in the force generating capacity of individual muscle cells independent of alpha motor neuron activation.

Keywords: anterior cruciate ligament; anterior cruciate ligament tear; muscle atrophy; muscle contractility; rehabilitation; isokinetic strength 


\section{Introduction}

Anterior cruciate ligament (ACL) tears are a frequent and debilitating injury, with rates in the US estimated to be up to 250,000 per year $^{1}$. Patients who tear their ACL have persistent quadriceps weakness of $20-40 \%$ or more, even after they have undergone ACL reconstruction (ACL-R) and returned to sport ${ }^{2-4}$. Several studies and reviews have suggested loss of proprioception, impaired neuromuscular control, and other neuroplastic changes as the primary causes of quadriceps weakness after ACL- $\mathrm{R}^{5-7}$. However, it is also possible that the whole muscle weakness observed after ACL tear occurs due to intrinsic changes within the muscle fibers themselves, as is observed in chronic rotator cuff disease and muscular dystrophy ${ }^{8,9}$. To gain a better understanding of the cellular mechanisms of muscle weakness in patients with ACL tears, we measured the force generating capacity of muscle fibers in vitro independent of the ability of motor neurons to activate these fibers, and hypothesized that ACL-R patients would reduce muscle fiber force production independent of the ability of motor neurons to activate these fibers.

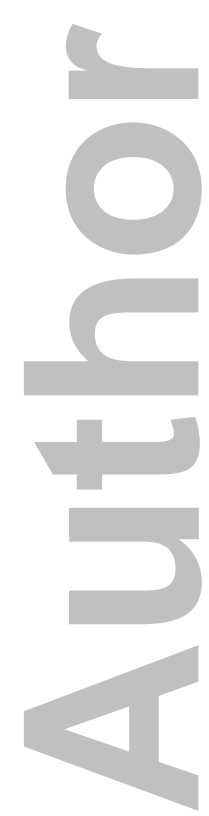




\section{Methods}

Subjects. This study was approved by the University of Michigan Institutional Review Board and conformed to the Declaration of Helsinki. Informed consent was obtained before enrollment. Eighteen to forty year old subjects with a unilateral complete ACL tear were eligible. Patients who were undergoing revision surgery, required a meniscal repair, had a previous injury to either knee, or had a myopathy or rheumatologic disorder were excluded. One week prior to surgery, patients performed strength measurements, and completed the International Knee Documentation (IKDC) survey, a patient reported outcomes assessment used to measure knee function $^{10}$. Normative IKDC values have been reported ${ }^{10}$. A biopsy of the vastus lateralis muscle was obtained at the time of surgery. Together these events were considered as the first study visit. Patients also completed the IKDC survey, underwent strength testing, and had a muscle biopsy performed at one month, two months, and six months ( \pm 1 week) after surgery.

Surgical Repair and Rehabilitation. Each patient sustained a tear within 12 weeks of surgery, and completed approximately 8 weeks of preoperative rehabilitation prior to surgical reconstruction. ACL reconstructions were performed by a single, fellowship-trained sports medicine surgeon using a hamstring tendon autograft and a single-bundle, anatomic reconstruction to recapitulate native ligament footprints and ligament obliquity. Patients participated in a standardized, accelerated post-operative rehabilitation program ${ }^{11}$. Approximately six months following repair, patients were discharged to full sport activities based on return to play criteria ${ }^{12}$.

Isokinetic Strength Measurements. Bilateral isokinetic strength measurements were performed in a System 3 dynamometer (BioDex, Shirley, NY) as described ${ }^{3}$. Briefly, isometric strength was measured at $90^{\circ}$ of knee flexion, and isokinetic extension strength was measured at 
$60^{\circ} / \mathrm{sec}$ over an $\operatorname{arc}$ of $90^{\circ}$ to $0^{\circ}$ of knee flexion. The highest force from five repetitions was used, and force data of the involved side was normalized to the uninjured side at each visit.

Muscle Biopsy. Biopsies were obtained from the vastus lateralis muscle. At the first visit, the biopsy was obtained intraoperatively, $3-5 \mathrm{~cm}$ proximal to the joint line using a percutaneous arthroscopic duckbill biter. Subsequent biopsies were obtained in the clinic under local anesthetic using a percutaneous biopsy needle as described ${ }^{13}, 3 \mathrm{~cm}$ proximal to the previous biopsy.

Muscle Fiber Contractility. Biopsies were prepared for muscle fiber contractility as described $^{9,14}$ (see supplementary material for details). Briefly, biopsies were placed in calcium chelating and cryoprotectant solutions, and stored at $-80^{\circ} \mathrm{C}$. For contractility testing, fibers were isolated from thawed bundles, and secured to a servomotor (Aurora Scientific, Aurora, ON) and force transducer (Aurora Scientific) using 10-0 nylon suture. Fiber length was adjusted to obtain a sarcomere length of $2.7 \mu \mathrm{m}$. Fiber cross sectional area (CSA) was calculated from width measurements that were fitted to an ellipse. Maximum isometric force $\left(\mathrm{F}_{\mathrm{o}}\right)$ was elicited by immersing the fiber in a high-calcium activation solution, and specific force $\left(\mathrm{sF}_{\mathrm{o}}\right)$ was determined by dividing $\mathrm{F}_{\mathrm{o}}$ by CSA. Ten to fifteen type II fibers were tested from each biopsy. Historical control data of uninjured subjects was reported previously ${ }^{14}$.

Statistical Analyses. Values presented are mean \pm SD. SigmaPlot 12.0 (Systat, San Jose, CA) was used for statistical analyses. Differences between time points were tested using a repeated measures one-way ANOVA $(\alpha=0.05)$ followed by Fisher's LSD post-hoc sorting. 


\section{Results}

There were 10 subjects, 5 males and 5 females (age 27.4 \pm 6.1 years and BMI 24.6 \pm 1.9 ). At the time of surgery, IKDC scores were $44 \%$ lower than uninjured controls (Figure 1). One month later, scores decreased by an additional $27 \%$, but by two months had returned to perioperative levels (Figure 1). Six months after surgery, scores were 29\% higher than at the start of the study, but were still $27 \%$ lower than normative data (Figure 1).

Isometric knee extension strength at the time of surgery was $13 \%$ lower in the injured limb than the uninjured limb (Figure $2 \mathrm{~A}$ ). One month later, there was a $68 \%$ decrease in relative strength compared to the perioperative value, and by two months relative strength was still reduced by $41 \%$ (Figure $2 \mathrm{~A}$ ). At six months, there was a $17 \%$ strength deficit compared to the time of surgery, and a $26 \%$ deficit compared to the uninjured limb (Figure 2A). Isokinetic strength generally changed in a similar pattern to isometric values (Figure 2B).

We next measured muscle fiber contractility (Figure 3). Perioperative CSA values were close to those from healthy, uninjured muscles, but decreased by $23 \%$ one month after surgery (Figure 3A). Thereafter, there were no significant differences in CSA. At the time of surgery, $\mathrm{F}_{\mathrm{o}}$ was $32 \%$ lower than uninjured muscles (Figure $3 \mathrm{~B}$ ). $\mathrm{F}_{\mathrm{o}}$ declined by an additional $34 \%$ at 1 month, and by two and six months was not different from the perioperative value (Figure 3B). At the time of discharge, $\mathrm{F}_{\mathrm{o}}$ remained $27 \%$ lower than controls (Figure $3 \mathrm{~B}$ ). $\mathrm{sF}_{\mathrm{o}}$ followed generally similar trends, displaying a 30\% reduction compared to control muscles at the time of surgery, and at the time of discharge remained 22\% lower than controls (Figure 3C). 


\section{Discussion}

Deficiencies in the ability of the nervous system to recruit and activate muscle fibers have been suggested as the primary factors behind the quadriceps muscle weakness in patients with ACL tears ${ }^{7}$. By measuring the contractility of muscle fibers in vitro, we decoupled muscle force generation from motor neuron input, and identified a muscle fiber-autonomous deficit in contractile function in patients with ACL tears.

Various mechanisms of neuromuscular deficiency have been proposed to explain weakness following ACL-R. ACL rupture causes remodeling of the central nervous system ${ }^{15}$ that affects both reflexive movement and voluntary movement in the injured limb ${ }^{7}$. One result of central nervous system remodeling is arthrogenic muscle inhibition, during which abnormal signals conveyed from mechanoreceptors or nociceptors in the knee joint after ACL tear inhibit the activation of alpha motor neurons, subsequently reducing muscle force production ${ }^{15}$. Much of the literature on neuromuscular changes in ACL-R patients has not considered intrinsic changes to the muscle fibers themselves. However, recent studies have pointed to clear evidence of maladaptive muscle responses, with ACL-R patients demonstrating increased circulating levels of the atrophy-inducing signaling molecules myostatin and TGF- $\beta$ up to 5 weeks after surgical repair $^{3}$, and decreased quadriceps muscle stem cell density and muscle fiber $\operatorname{CSA}^{16,17}$. This is further supported by animal studies that reported reduced in vitro whole muscle force production after ACL tear, with increased expression of atrophy-associated genes such as the E3 ubiquitin ligases atrogin-1, MuRF-1, and MUSA-1 that can target proteins for enzymatic breakdown ${ }^{18,19}$. In the current study, the lack of appreciable changes in fiber CSA along with substantial changes in $\mathrm{F}_{0}$, suggest that there is a marked reduction in the density of myofibrils, which are the force generating organelles in muscle fibers. Although speculative, the results of the current 
manuscript and previous studies suggest that it is possible that after ACL tear and surgical reconstruction there is an initial activation of proteolytic processes within fibers that lead to a reduction in force production through loss of myofibrils. Subsequent to this bout of atrophy, during the rehabilitation period there is then likely insufficient activation of muscle stem cells and protein synthesis signaling pathways to fully restore the myofibrils lost due to the tear and surgical repair, resulting in overall persistent muscle weakness in ACL-R patients.

While we did not measure histological or biochemical changes in biopsied tissue, or contractility beyond 6 months after surgery, our study provided insight into the changes within muscle independent of neural input after ACL tear and reconstruction. Studying the pathological changes to muscle fibers is important, as the muscle weakness which results after ACL tear reduces athletic performance, and likely contributes to the development of osteoarthritis ${ }^{2,20}$. Many ACL rehabilitation programs put substantial focus on neuromuscular re-education, but more emphasis on exercises for muscle hypertrophy may be beneficial. Additionally, studies that evaluate the ability of pharmacological interventions to prevent myofibrillar protein degradation in the acute post-operative period, or increase myofibrillar protein synthesis in the long term, are likely warranted. 


\section{Abbreviations}

ACL, Anterior cruciate ligament

ACL-R, Anterior cruciate ligament reconstruction

CSA, Cross-sectional area

$\mathrm{F}_{\mathrm{o}}$, Maximum isometric force

IKDC, International knee documentation committee

$\mathrm{sF}_{\mathrm{o}}$, Specific force, maximum isometric force normalized to cross-sectional area

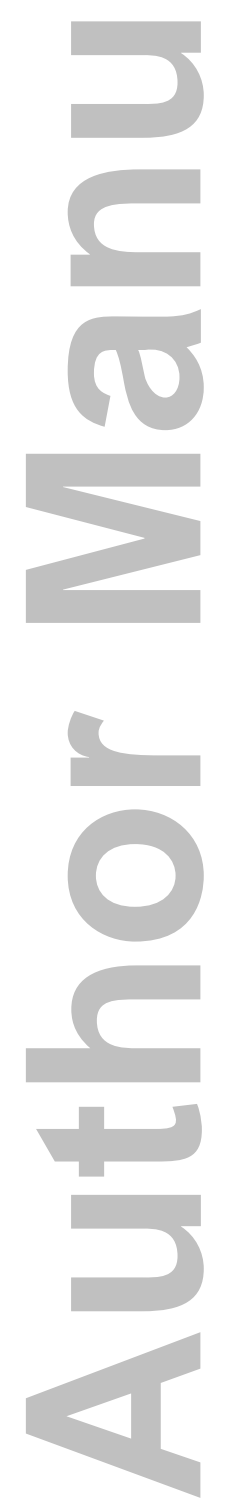

John Wiley \& Sons, Inc. 


\section{References}

1. Griffin LY, Albohm MJ, Arendt EA, et al. Understanding and preventing noncontact anterior cruciate ligament injuries: a review of the Hunt Valley II meeting, January 2005. Am J Sports Med 2006;34:1512-1532.

2. Palmieri-Smith RM, Thomas AC, Wojtys EM. Maximizing quadriceps strength after ACL reconstruction. Clin Sports Med 2008;27:405-424.

3. Mendias CL, Lynch EB, Davis ME, et al. Changes in circulating biomarkers of muscle atrophy, inflammation, and cartilage turnover in patients undergoing anterior cruciate ligament reconstruction and rehabilitation. Am J Sports Med 2013;41:1819-1826.

4. Ingersoll CD, Grindstaff TL, Pietrosimone BG, Hart JM. Neuromuscular consequences of anterior cruciate ligament injury. Clin Sports Med 2008;27:383-404.

5. Brown TN, Palmieri-Smith RM, McLean SG. Sex and limb differences in hip and knee kinematics and kinetics during anticipated and unanticipated jump landings: implications for anterior cruciate ligament injury. Br J Sports Med 2009;43:1049-1056.

6. Williams GN, Snyder-Mackler L, Barrance PJ, Buchanan TS. Quadriceps femoris muscle morphology and function after ACL injury: a differential response in copers versus noncopers. J Biomech 2005;38:685-693.

7. Ward S, Pearce AJ, Pietrosimone B, Bennell K, Clark R, Bryant AL. Neuromuscular deficits after peripheral joint injury: a neurophysiological hypothesis. Muscle Nerve 2015;51:327-332.

8. Gumucio JP, Davis ME, Bradley JR, et al. Rotator cuff tear reduces muscle fiber specific force production and induces macrophage accumulation and autophagy. J Orthop Res 2012;30:1963-1970.

9. Mendias CL, Roche SM, Harning JA, et al. Reduced muscle fiber force production and disrupted myofibril architecture in patients with chronic rotator cuff tears. J Shoulder Elbow Surg 2015;24:111-119.

10. Anderson AF, Irrgang JJ, Kocher MS, Mann BJ, Harrast JJ, Committee I. The International Knee Documentation Committee Subjective Knee Evaluation Form: normative data. Am J Sports Med 2006;34:128-135.

11. Beynnon BD, Uh BS, Johnson RJ, et al. Rehabilitation after anterior cruciate ligament reconstruction: a prospective, randomized, double-blind comparison of programs administered over 2 different time intervals. Am J Sports Med 2005;33:347-359.

12. Beynnon BD, Johnson RJ, Abate JA, Fleming BC, Nichols CE. Treatment of anterior cruciate ligament injuries, part 2. Am J Sports Med 2005;33:1751-1767.

13. Sarver DC, Sugg KB, Disser NP, Enselman ERS, Awan TM, Mendias CL. Local 
cryotherapy minimally impacts the metabolome and transcriptome of human skeletal muscle. Sci Rep 2017;7:2423.

4. Claflin DR, Larkin LM, Cederna PS, et al. Effects of high-and low-velocity resistance training on the contractile properties of skeletal muscle fibers from young and older humans. J Appl Physiol 2011;111:1021-1030.

5. Palmieri-Smith RM, Thomas AC. A neuromuscular mechanism of posttraumatic osteoarthritis associated with ACL injury. Exerc Sport Sci Rev 2009;37:147-153.

6. Noehren B, Andersen A, Hardy P, et al. Cellular and Morphological Alterations in the Vastus Lateralis Muscle as the Result of ACL Injury and Reconstruction. J Bone Joint Surg Am 2016;98:1541-1547.

7. Fry CS, Johnson DL, Ireland ML, Noehren B. ACL injury reduces satellite cell abundance and promotes fibrogenic cell expansion within skeletal muscle. J Orthop Res. December 2016. doi:10.1002/jor.23502.

8. Wurtzel CN, Gumucio JP, Grekin JA, et al. Pharmacological inhibition of myostatin protects against skeletal muscle atrophy and weakness after anterior cruciate ligament tear. JOrthop Res 2017 (to be published).

9. Delfino GB, Peviani SM, Durigan JLQ, et al. Quadriceps muscle atrophy after anterior cruciate ligament transection involves increased mRNA levels of atrogin-1, muscle ring finger 1, and myostatin. Am J Phys Med Rehabil 2013;92:411-419.

0. Keays SL, Newcombe PA, Bullock-Saxton JE, Bullock MI, Keays AC. Factors involved in the development of osteoarthritis after anterior cruciate ligament surgery. Am J Sports Med 2010;38:455-463.

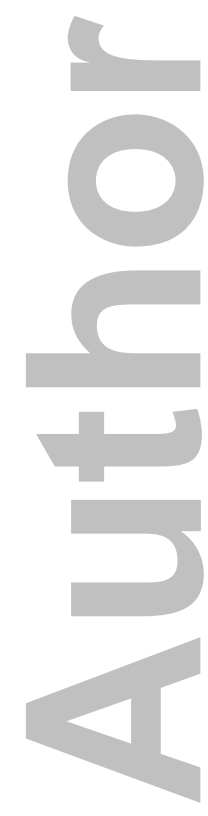




\section{Figure Legends}

Figure 1. International Knee Documentation Committee (IKDC) scores. Values are mean \pm SD. Horizontal dashed line indicates the mean normative value previously published ${ }^{10}$. Differences tested with a one-way repeated measures ANOVA $(\alpha=0.05)$ followed by Fisher's LSD post hoc sorting.

Figure 2. Strength Measurements. (A) Maximum isometric extension strength, and (B) maximum isokinetic knee extension strength. The injured side was normalized to the uninjured side at each time point to generate relative values. Horizontal dashed line indicates bilateral strength symmetry. Values are mean \pm SD. Differences tested with a one-way repeated measures ANOVA $(\alpha=0.05)$ followed by Fisher's LSD post hoc sorting.

Figure 3. Permeabilized fiber contractility. (A) Cross-sectional area (CSA), (B) maximum isometric force $\left(\mathrm{F}_{\mathrm{o}}\right)$, and $(\mathrm{C})$ specific force $\left(\mathrm{sF}_{\mathrm{o}}, \mathrm{F}_{\mathrm{o}}\right.$ normalized by CSA). Values are mean \pm SD. Horizontal dashed line indicates the mean normative value from uninjured, healthy, similar age subjects $(24.5 \pm 2.5$ years of age) previously published, using the same equipment and techniques as the current study ${ }^{14}$. Differences tested with a one-way repeated measures ANOVA $(\alpha=0.05)$ followed by Fisher's LSD post hoc sorting.

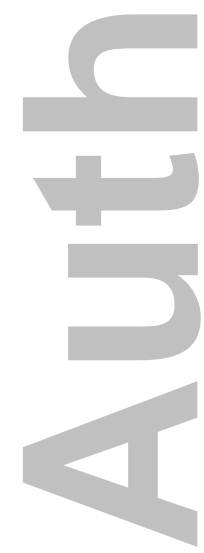


Page 13 p 6055 Muscle \& Nerve
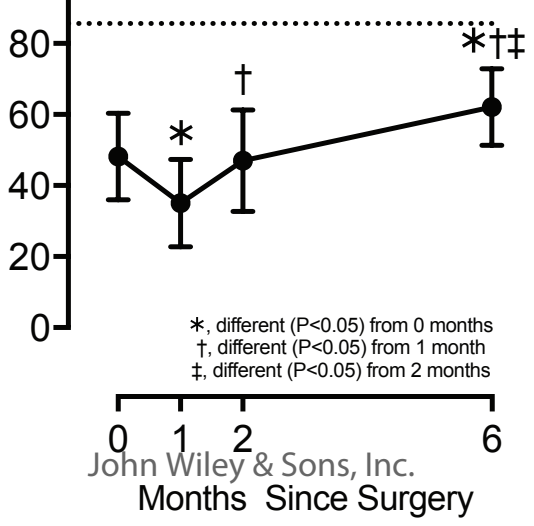

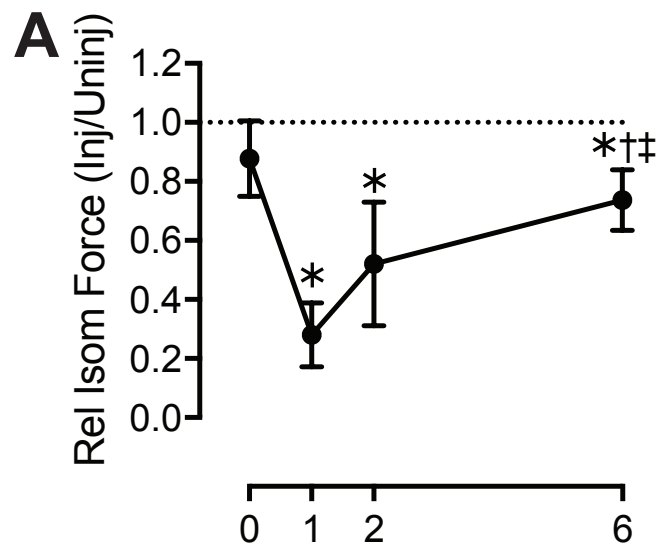

B
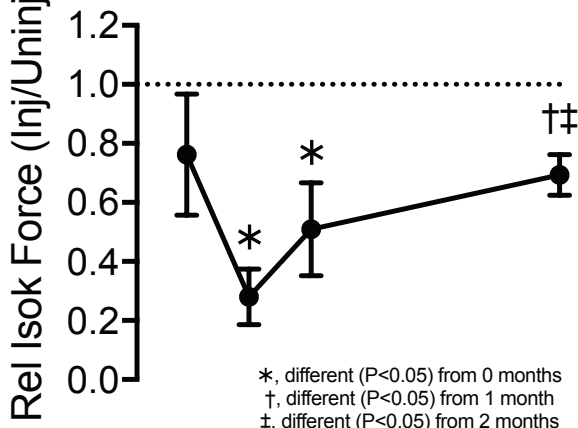

Months Since Surger\% Wiley \& Sons, Inc.

Months Since Surgery

This article is protected by copyright. All $r$ 

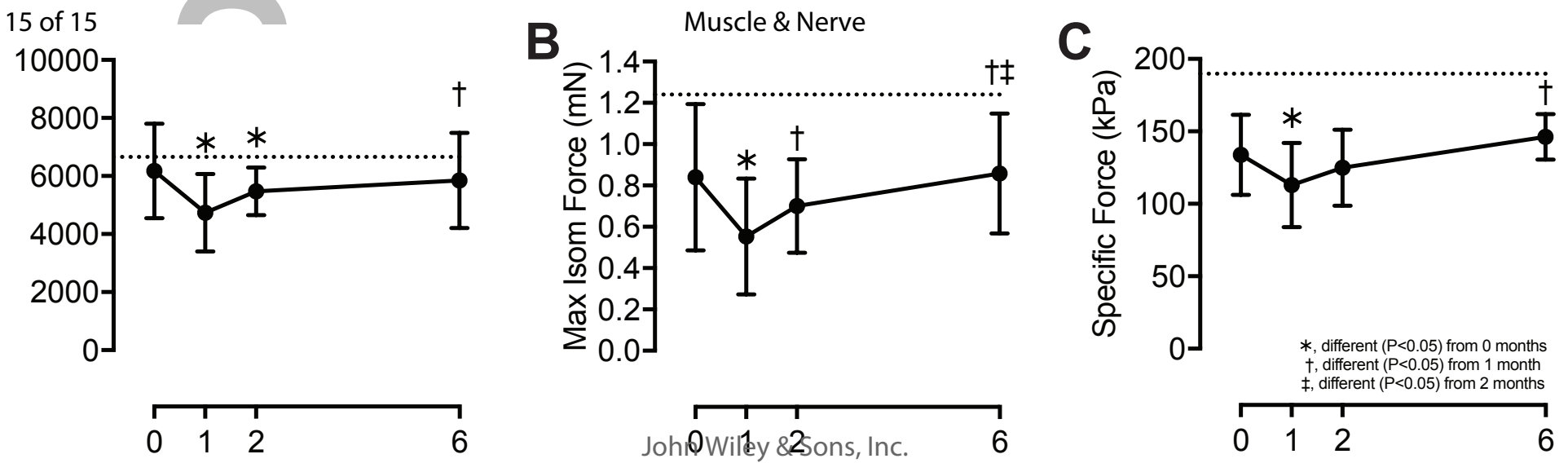

Joh@Wiley \&25ons, Inc. 6

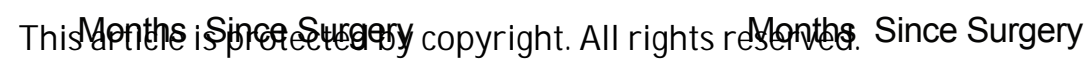

